\title{
Serum Leptin Levels in Patients with Hepatitis B: A Meta-analysis
}

\author{
Qiong-yuan ZHANG ${ }^{1}$, Xuan XU' ${ }^{2}$, Mei LUO ${ }^{2}$, Jian-jiang XUE ${ }^{1, a,{ }^{*}}$ and \\ Ying-li $\mathrm{LI}^{2, \mathrm{~b}}$ \\ ${ }^{1}$ Department of Clinical Laboratory, The University-Town Affiliated Hospital of \\ Chongqing Medical University, Chongqing 401331, China. \\ ${ }^{2}$ School of Public Health and Management, Chongqing Medical University, Chongqing, \\ China, 400016 \\ ajianjiangxue@126.com, bliylyl@126.com \\ ${ }^{*}$ Corresponding author
}

Keywords: Leptin, Hepatitis B virus, Male, Female, Meta-analysis.

\begin{abstract}
Background and Aims: Hepatitis B virus (HBV) infection is a widespread disease affecting people worldwide. With more and more research on the deepening of the leptin, which suggested a close relationship between serum leptin levels and HBV. In this study, we explored the correlation between serum leptin levels and HBV through meta-analysis.

Methods: Potentially relevant studies between 2007 and 2017 were retrieved from PubMed, Ovid, Embase, Web of Science, Weipu Journal, Chinese Biomedical, China National Knowledge Infrastructure, and Wan Fang Data. Z-test was used to determine the significance of the pooled standardized mean difference (SMD).

Results: We analyzed 16 studies, which involved $884 \mathrm{HBV}$ patients and 618 healthy controls. And then pooled the SMD and calculated the 95\% confidence interval (CI) by using a random-effects model from heterogeneity analysis. High serum leptin levels were observed in HBV patients (SMD: 1.16, 95\%CI: 0.72-1.59, P<0.00001). Stratification analysis on the ethnicity, study design, and detection method revealed significant increases in the serum leptin levels among the Asian population of the ethnicity subgroup, case-control study of the study design subgroup, and ELISA or RIA of the detection method subgroup.

Conclusion: This meta-analysis suggests a significant difference between HBV patients and healthy controls. Therefore, serum leptin levels may serve as a prognostic marker for hepatitis B disease.
\end{abstract}

\section{Introduction}

Leptin is a protein hormone secreted by adipose and is composed of 167 amino acids [1]. In 1994, Zhang et al. [2] applied the method of directional cloning from obesity and abnormal glucose tolerance in mice to get its obesity gene, and the homologous sequences of human beings for the first time. Leptin has been found to play an important role in the hypothalamic metabolism-regulation center in many studies, which suppresses appetite, increases energy consumption, and influences fat synthesis and endocrine functions [3]. As a proinflammatory cytokine, leptin is involved in the modulation of the immune response [4].

Hepatitis B virus (HBV) infection is a worldwide public health problem. The World Health Organization reported that two billion people suffer from HBV infection worldwide, and 240 million people are affected by chronic HBV infection [5, 6]. 
Furthermore, HBV can lead to liver failure [7], liver cirrhosis, and hepatocellular carcinoma (HCC)[8]. It is showing that HBV is a crucial risk factor for liver diseases and a serious threat to human health. Thus HBV deserves considerable attention.

Hepatitis B is caused by hepatitis B virus infection, the diagnosis mainly depends on the serum HBV markers detection. Some studies have reported an increase in serum leptin levels in HBV infection because of the action of inflammatory cytokines, adipose tissues, and phagocytosis of macrophages. In addition, leptin stimulates macrophages to secrete inflammatory cells, such as TNF- $\alpha$, IL-6, and IL-12, which can promote liver degeneration and necrosis [9]. However, no differences in serum leptin levels were observed between HBV patients and healthy people in some studies [10]. Thus, we conducted this meta-analysis to estimate the association of serum leptin levels with both HBV patients and healthy controls.

\section{Materials and Methods}

\section{Search Strategy}

To identify all potentially relevant studies that investigated the association of serum leptin levels with HBV, we performed a systematic search in PubMed, Ovid, Embase, and Web of Science. In addition, the following Chinese databases were included: Weipu Journal, Chinese Biomedical, China National Knowledge Infrastructure, and WanFang Data. The following search terms were used: (Leptin or Lep or ob gene product or ob protein or obese gene product or obese protein) and (HBV or hepatitis B or hepatitis B virus or chronic hepatitis B or CHB). No language or publication status restriction was imposed.

\section{Inclusion Criteria}

The included articles satisfied the following criteria: (1) case-control, clinical cohort, or cross-sectional studies examining serum leptin levels between HBV patients and healthy controls; (2) detailed mean and standard deviation (SD) data of the serum leptin levels; and (3) publication year from 2007 to 2017. When a study was published more than once, all the data in the duplicate article were abstracted, and only one version of the articles was included in our study.

\section{Exclusion Criteria}

Studies with the following criteria were excluded: (1) contained conference abstracts, case reports, reviewed articles, and letter to the editor; (2) no reference to HBV and serum leptin levels; and (3) duplicated reports or studies on animals.

\section{Study Quality and Data Extraction}

Two reviewers independently reviewed and extracted the articles and assessed the methodological quality of the studies included by using the Newcastle-Ottawa Scale (NOS)[11]. The NOS scores ranged from 0 to 9, and a study was considered to present good quality at a score of $\geq 7$. A standardized data form was used to collect the following descriptive information from the included studies: first author, publication year, journal, source country, language, study design, number of cases and controls, detection method, and data of leptin values (mean \pm SD). Disagreements with regard to the data abstraction of the included studies were settled through discussion or reviewed by a third reviewer. 


\section{Statistical Analysis}

Statistical analysis was performed using Review Manager5.3. The pooled SMD with 95\% CI was calculated to evaluate the association between HBV patients and healthy individuals, and Z-test was used to determine the significance of the pooled SMD. Statistical heterogeneity within studies was evaluated using Cochran's Q test and $I^{2}$ test [12]. A fixed-effects model was used to determine whether heterogeneity exists among the studies $\left(\mathrm{P}>0.05\right.$ or $\left.I^{2}<50 \%\right)[13]$. Otherwise, a random-effects model was used. Sensitivity analysis was conducted by omitting the studies sequentially to evaluate the influence of single study on the overall estimate [14]. Additionally, Begg's test was performed using Stata/SE to detect potential publication bias [15].

\section{Results}

\section{Literature Search}

A total of 567 potential relevant publications until 2017 were systematically identified through an electronic database search. After excluding the duplicates, the retrieved studies were screened on the basis of the title and abstract for relevance. Studies without HBV, animal tests, case reports, summaries, meta-analysis, and conferences were excluded. Among the screened studies, four were excluded via full-text screening because they contain irrelevant data. Overall, 16 studies were included in our meta-analysis. The flow chart of the article selection process is shown in Fig.1.

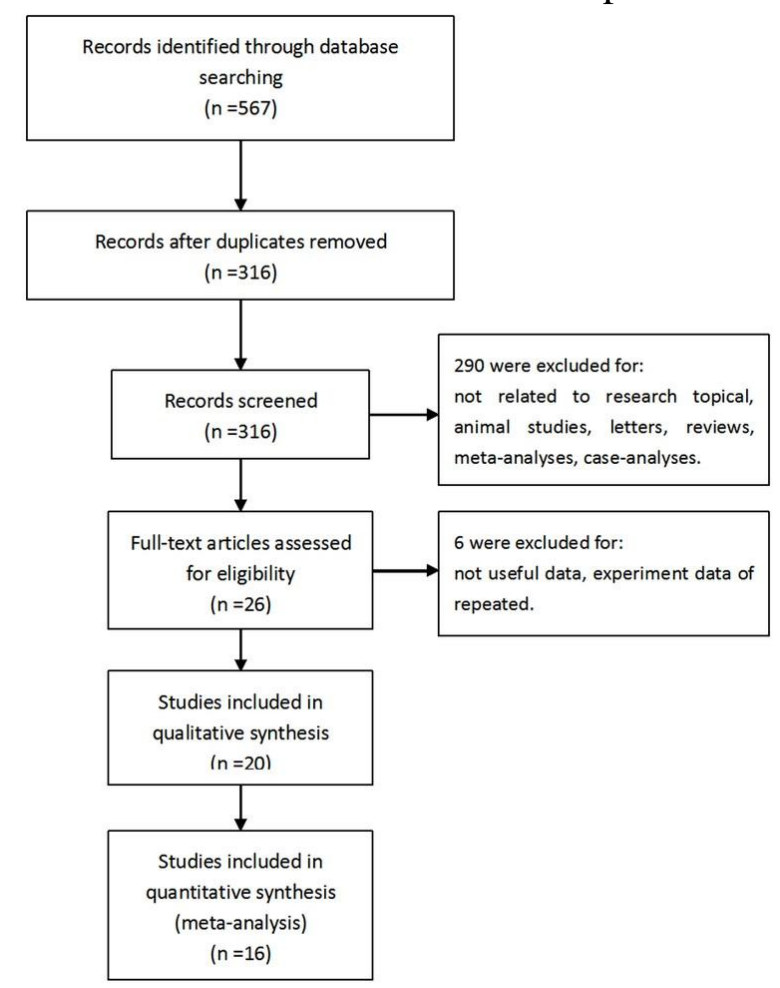

Fig.1. Flowchart of the selection process.

\section{Study Characteristics}

All eligible studies were compared. In these studies, 884 patients who had undergone HBV and 618 controls were incorporated into the current meta-analysis. The studies were published from 2007 to 2017. With regard to demographic variables, all of studies were conducted on an Asian population [10, 16-29], except one study for Caucasian [30]. With regard to the detection methods of the serum leptin levels, 2 
studies utilized a radioimmunoassay (RIA), and 14 studies performed an enzyme-linked immunosorbent assay (ELISA). With regard to the study design, one article presented a cross-sectional study and the rest were presented case-control studies. The basic characteristics of the studies are summarized in Table 1.

Table 1. The basic characteristics of the studies.

\begin{tabular}{llllllll}
\hline \multirow{2}{*}{ Author } & Pubication & Ethnicity & Study design & Method & Total Sample & NOS \\
\cline { 5 - 7 } & year & & & & Case & Control & score \\
\hline Chingsheng Hsu & 2015 & Asian & cross-sectional & ELISA & 187 & 184 & 7 \\
Chun Zhao & 2008 & Asian & case-control & ELISA & 107 & 17 & 6 \\
Daorong Jiang & 2009 & Asian & case-control & ELISA & 39 & 15 & 6 \\
Handong Li & 2007 & Asian & case-control & ELISA & 28 & 16 & 6 \\
Hui Tian & 2015 & Asian & case-control & ELISA & 62 & 50 & 6 \\
Jiangao Li & 2012 & Asian & case-control & RIA & 35 & 30 & 6 \\
Jing Yang & 2014 & Asian & case-control & ELISA & 65 & 50 & 6 \\
Naiguo Li & 2009 & Asian & case-control & ELISA & 36 & 30 & 6 \\
Qinghua Yu & 2014 & Asian & case-control & ELISA & 52 & 46 & 6 \\
Xiaoqi Huang & 2007 & Asian & case-control & RIA & 43 & 38 & 6 \\
Xiping Guo & 2008 & Asian & case-control & ELISA & 19 & 12 & 6 \\
Xiuzhen Zhang & 2012 & Asian & case-control & ELISA & 42 & 20 & 6 \\
Yan Su & 2012 & Asian & case-control & ELISA & 45 & 30 & 6 \\
Yingyan Lu & 2013 & Asian & case-control & ELISA & 65 & 25 & 6 \\
Zerrin AŞÇI & 2012 & Caucasian & case-control & ELISA & 39 & 35 \\
Zhihua Yang & 2016 & Asian & case-control & ELISA & 20 & 20 & 6 \\
\hline
\end{tabular}

\section{Quantitative Data Synthesis}

In this meta-analysis, the HBV patients and healthy controls were analyzed using a random-effects model to determine the heterogeneity $\left(\mathrm{P}<0.00001, I^{2}=93 \%\right)$. The results showed a significant heterogeneity between HBV patients and healthy controls. The included studies were then classified into subgroups by ethnicity (Asian population subgroup, $\mathrm{P}<0.00001, I^{2}=93 \%$ ), by study design (case-control study subgroup, $\mathrm{P}<0.00001, I^{2}=84 \%$ ), and by detection method (ELISA subgroup, $\mathrm{P}<0.00001, I^{2}=93 \%$; RIA subgroup, $\mathrm{P}=0.005, I^{2}=87 \%$ ). The results revealed statistical significant differences between HBV patients and healthy controls with regard to serum leptin levels. Compared with healthy individuals, HBV patients presented higher serum leptin levels according to the results of random-effects analysis, which pooled the SMD and 95\%CI (SMD: 1.16, 95\%CI: 0.72-1.59, P<0.00001) (Fig.2). Ethnicity-stratified analysis results showed that HBV patients were associated with increased serum leptin levels in contrast to the healthy controls in the Asian population subgroup (SMD: 1.16, 95\%CI: 0.69-1.62, P<0.00001) (Fig.3). High serum leptin levels were observed in the case-control study subgroup according to the design-stratified subgroup analysis (SMD: 1.25, 95\%CI: 0.91-1.59, P<0.00001) (Fig.4). Furthermore, in the detection method-stratified subgroup analysis results, HBV patients in the ELISA subgroup presented high serum leptin levels (SMD: 1.16, 95\%CI: $0.67-1.64, \mathrm{P}<0.00001$ ), and a similar correlation was observed in the RIA subgroup (SMD: 1.17, 95\%CI: 0.14-2.20, P=0.03) (Fig.5). 


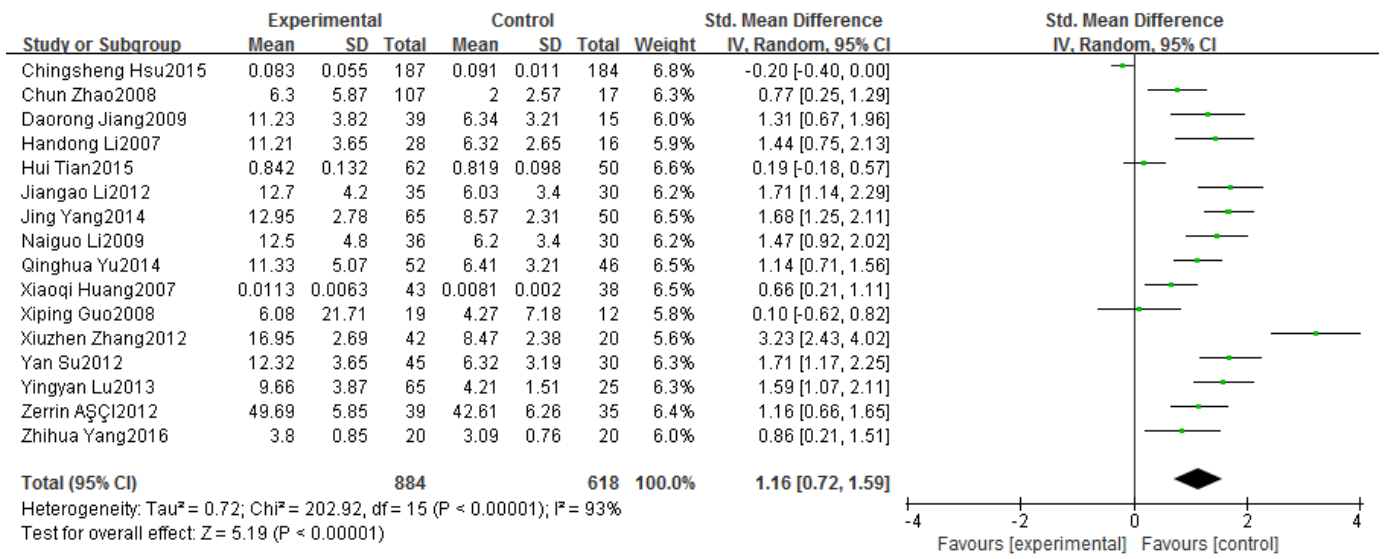

Fig.2. The forest plot for the association between serum leptin levels and healthy controls.

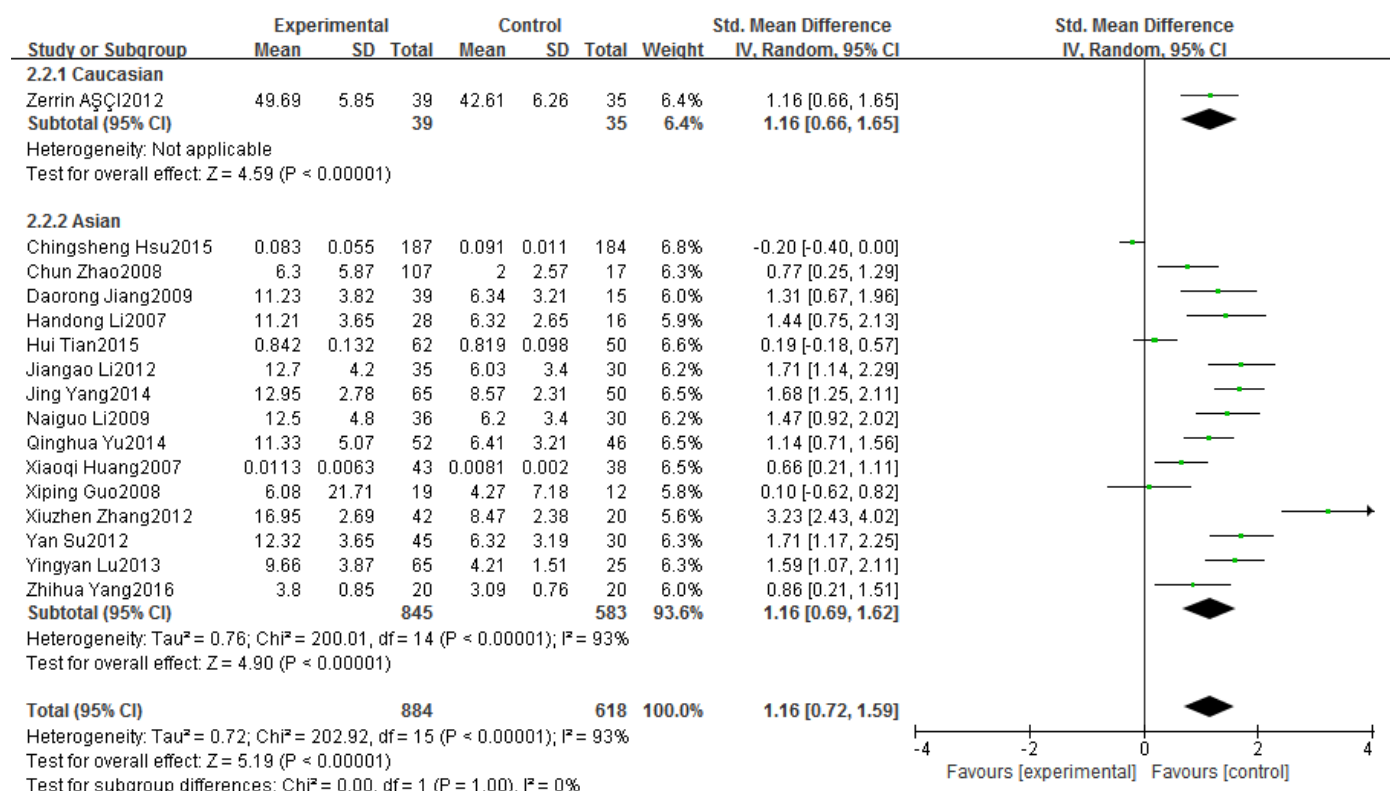

Fig.3. The forest plot for the association between serum leptin levels and healthy controls in the subgroup analysis by different ethnicity.

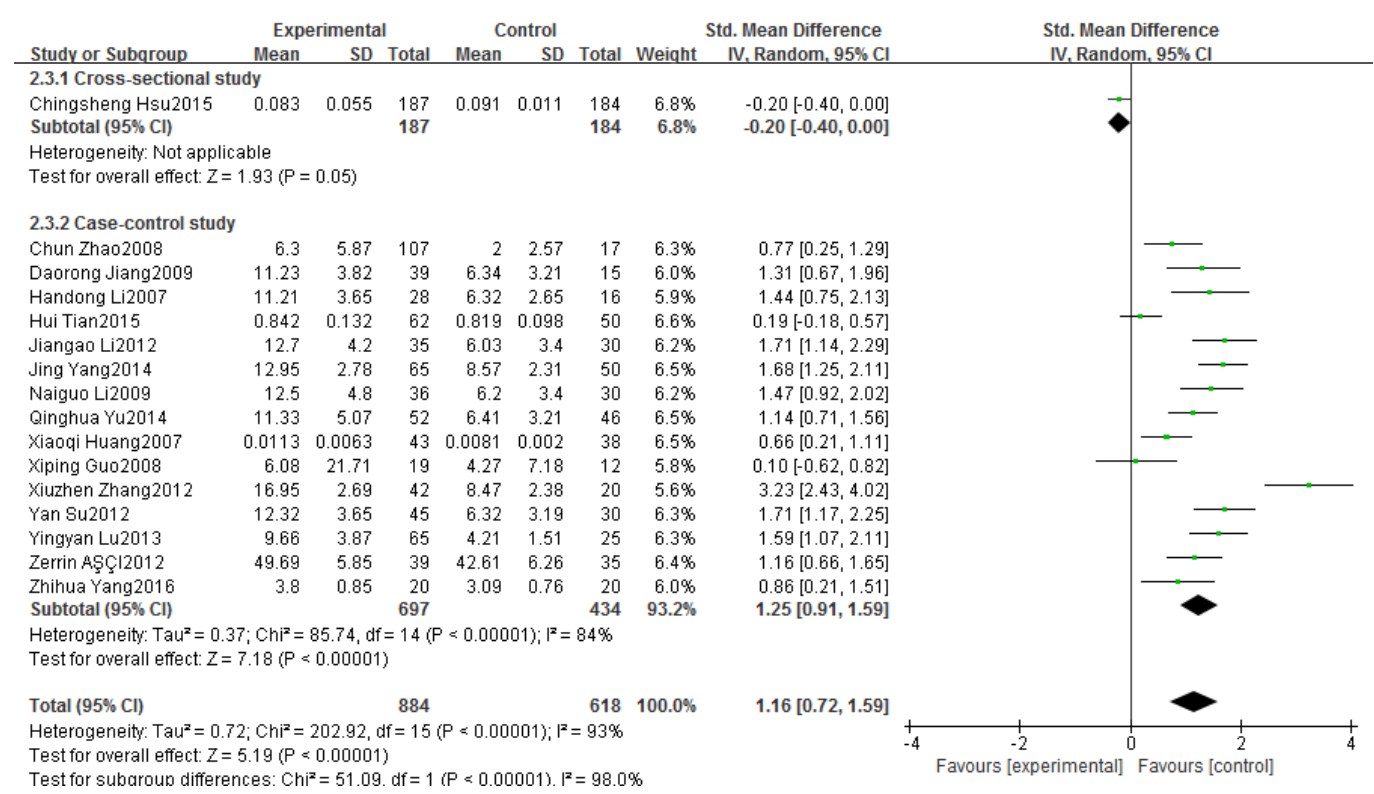

Fig.4. The forest plot for the association between serum leptin levels and healthy controls in the subgroup analysis by different study design. 


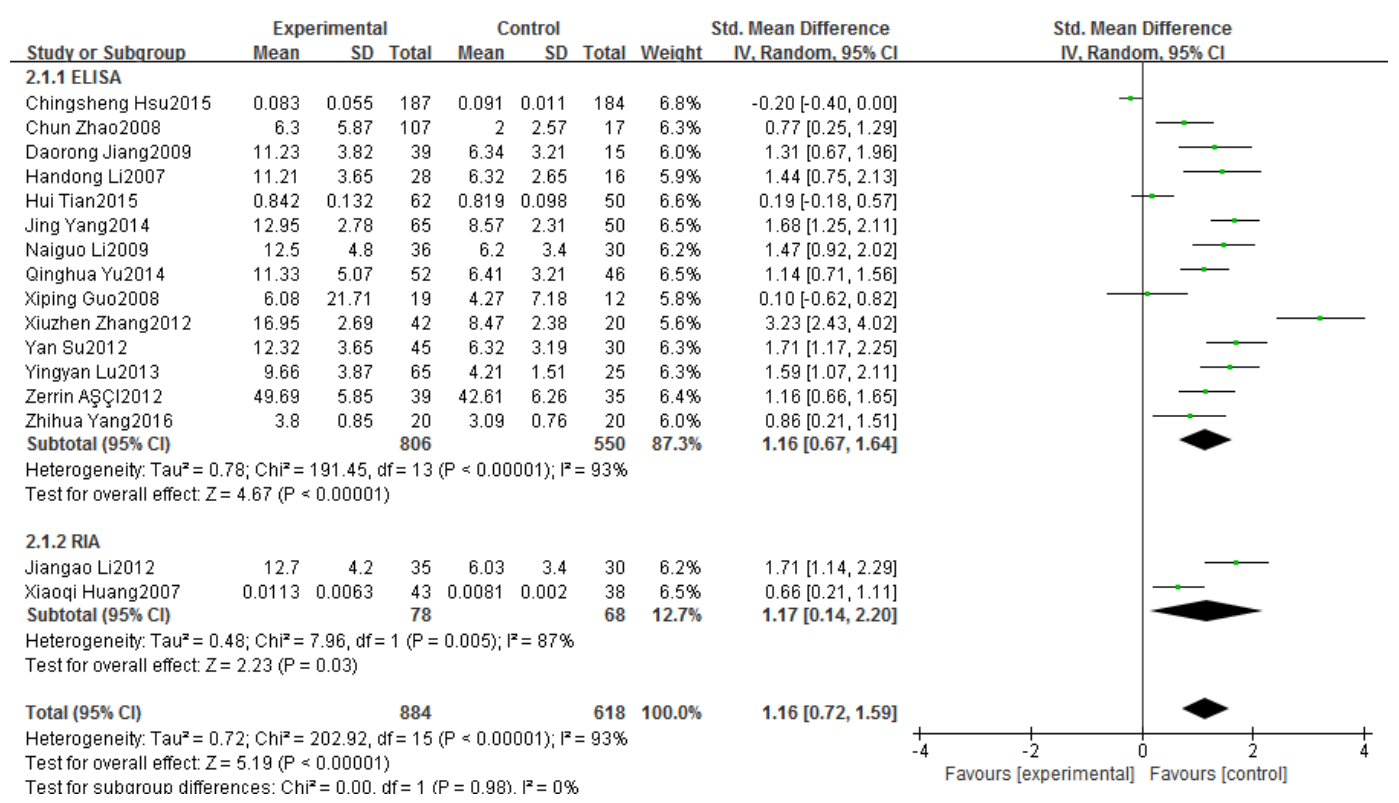

Fig.5. The forest plot for the association between serum leptin levels and healthy controls in the subgroup analysis by different detection method.

\section{Sensitivity Analyses and Publication Bias}

Sensitivity analyses were conducted by omitting individual studies sequentially. The results of the pooled SMD indicated that no material change in the meta-analysis. In some conditions, the $\mathrm{P}$ value or $I^{2}$ value for the heterogeneity decreased. Publication bias among the studies was evaluated using Begg's test, and the results indicated a symmetrical distribution of the funnel plot, indicating no publication bias between the serum leptin levels of the HBV patients and those of healthy controls $(\mathrm{P}=0.224>0.05)$.

\section{Discussion}

HBV is a clinically common disease. Thus, one of the main objectives of clinical research on HBV is to obtain a clear and reliable result that can be used as a basis for clinical diagnosis in patients. Numerous studies have explored the relationship between serum leptin levels and HBV. However, such clinical trials did not yield reliable and consistent results. For many individual studies, the sample was extremely small, and the results were inconclusive. A meta-analysis circumvents the limits of a single sample clinical trial and produces comprehensive and reliable results, which can serve as a good basis for medical decisions in patients.

In recent years, an increasing number of clinical trials have focused on the relationship between leptin and HBV. As an inflammatory mediator, leptin can affect various physiological processes and immune responses and can mediate $\mathrm{T}$ cells immune to hepatitis [31]. In this meta-analysis, we retrieved 16 studies to investigate the association between serum leptin levels and HBV. Our data showed a significant difference between HBV patients and healthy controls. And some studies which were included showed that serum leptin levels in females were higher than those in males (Fig.6). 


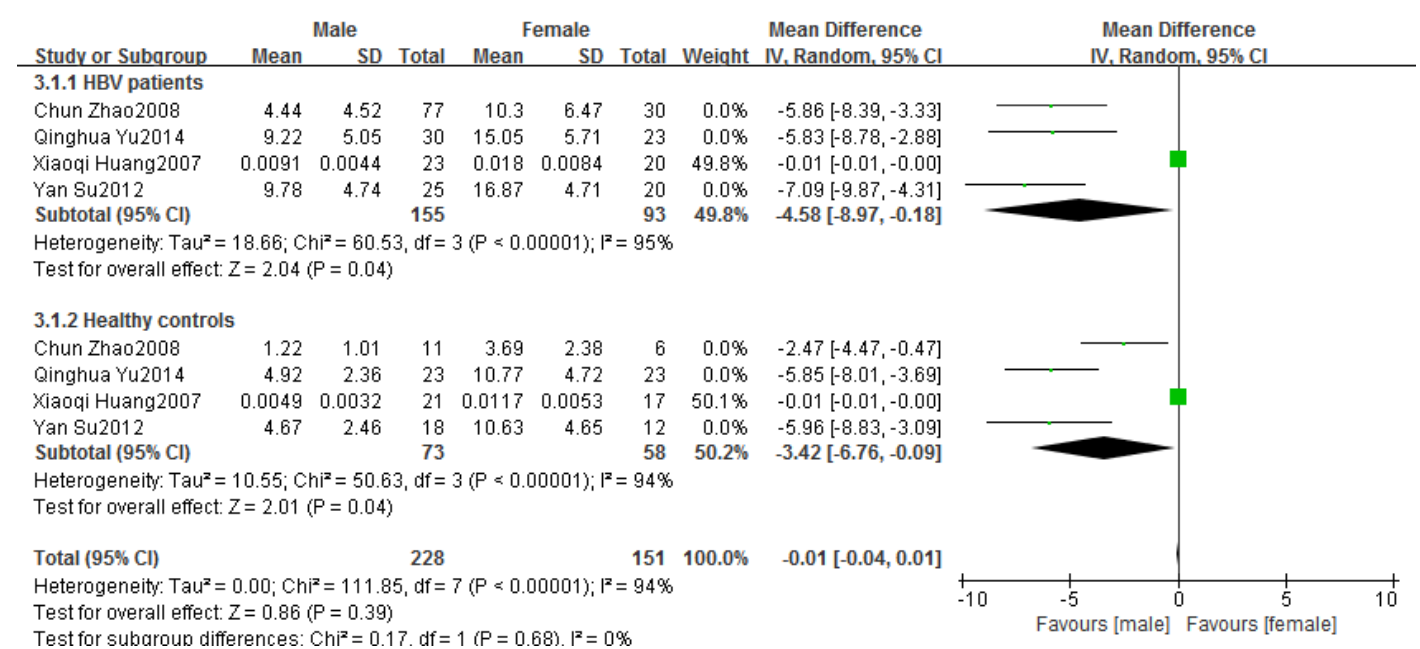

Fig.6. The forest plot presenting the quantitative synthesis of serum leptin levels for comparing the groups of HCV patients; male vs. female, healthy controls; male vs. female.

Several limitations were also considered in the present study. First, we detected apparent heterogeneity in the included studies, which were selected strictly by inclusion criteria. All included studies were conducted on different areas; thus, their diagnoses were different, and their eligible patients presented significant individual differences. Meanwhile, partial studies were not homogeneous with BMI, age, and other influencing factors between the HBV patients and healthy controls. Therefore, the heterogeneity among the included studies was high. Second, studies with positive results were more likely to be accepted by journals than those with negative results. To some extent, the lack of negative results could have affected the results of the present study [32]. Third, most studies included used a small sample size of HBV patients and healthy controls. Hence, the results of these studies that would be used in the meta-analysis must be interpreted cautiously. Fourth, the approximation numbers were in partial data. Thus, it may be biased compared with the actuality. Fifth, we included only three studies on Caucasian population; thus, the data on Caucasian subjects were inadequate.

Overall, the results of this meta-analysis represent the most robust estimate for the effect of serum leptin levels on HBV patients, showing that a high level of serum leptin is associated with HBV. In addition, this meta-analysis warrants further clinical investigations with well-designed and large case-control studies for the identification of the role of leptin in the development of HBV.

\section{References}

[1] Marwarha, G. and O. Ghribi, Leptin signaling and Alzheimer's disease. Am J Neurodegener Dis, 2012. 1(3): p. 245-65.

[2] Zhang, Y., et al., Positional cloning of the mouse obese gene and its human homologue. Nature, 1994, 372(6505): p. 425-32.

[3] Fantuzzi, G., Adipose tissue, adipokines, and inflammation. J Allergy Clin Immunol, 2005. 115(5): p. 911-9; quiz 920.

[4] Procaccini, C., et al., Leptin as immune mediator: Interaction between neuroendocrine and immune system. Dev Comp Immunol, 2017, 66: p. 120-129. 
[5] Dienstag, J.L., Hepatitis B virus infection. N Engl J Med, 2008. 359(14): p. 1486-500.

[6] Guidelines for the Prevention, Care and Treatment of Persons with Chronic Hepatitis B Infection. WHO Guidelines Approved by the Guidelines Review Committee. 2015, Geneva: World Health Organization.

[7] Shen, Y., et al., A comprehensive validation of HBV-related acute-on-chronic liver failure models to assist decision-making in targeted therapeutics. Sci Rep, 2016. 6: p. 33389.

[8] Yin, J.W., H.M. Ping and B. Zhong, Intrahepatic Toll-Like Receptor 3 in Chronic HBV Infection Subjects: Asymptomatic Carriers, Active Chronic Hepatitis, Cirrhosis, and Hepatocellular Carcinoma. Hepat Mon, 2016, 16(6): p. e34432.

[9] Sennello, J.A., et al., Regulation of $\mathrm{T}$ cell-mediated hepatic inflammation by adiponectin and leptin. Endocrinology, 2005, 146(5): p. 2157-64.

[10]Hsu, C.S., et al., Adipocytokines and liver fibrosis stages in patients with chronic hepatitis B virus infection. Hepatol Int, 2015. 9(2): p. 231-42.

[11] Stang, A., Critical evaluation of the Newcastle-Ottawa scale for the assessment of the quality of nonrandomized studies in meta-analyses. Eur J Epidemiol, 2010. 25(9): p. 603-5.

[12]Higgins, J.P. and S.G. Thompson, Quantifying heterogeneity in a meta-analysis. Stat Med, 2002, 21(11): p. 1539-58.

[13]Higgins, J.P., et al., Measuring inconsistency in meta-analyses. BMJ, 2003, 327(7414): p. 557-60.

[14] Whitlock, R.P., et al., Clinical benefit of steroid use in patients undergoing cardiopulmonary bypass: a meta-analysis of randomized trials. Eur Heart J, 2008. 29(21): p. 2592-600.

[15]Egger, M., et al., Bias in meta-analysis detected by a simple, graphical test. BMJ, 1997, 315(7109): p. 629-34.

[16] Yang, Z., F. Shen and H. Huang, The change and clinical significance of serum leptin levels in chronic liver disease patients, Contemporary Med, 2016. 22(4): p. 82-83.

[17] Tian, H. and Z. He. Discuss about chronic HBV infection status and replication correlation with serum letin and adiponectin levels. Chin J Mod Drug Appl, 2015. 9(14): p. 33-35.

[18] Yang, J. and C. Huang, Correlation of Liver Function and Viral Replication with Lipid Metabolism in Patients with Hepatitis B and NAFLD. J of Kunming Med Univ, 2014, 35(5): p. 111-113.

[19] Yu, Q., et al., Signifi cance of adiponectin and leptin levels in patients with chronic hepatitis B. Chin J Liver Dis(Electr Version), 2014. 6(4): p. 19-21.

[20]Lu, Y., et al., Association of serum leptin levels with hepatic fibrosis in chronic viral hepatitis. Zhejiang Med J, 2013, 35(12): p. 1150-1152. 
[21]Li, J., et al., Clinical Significance of Changes on Serum Levels of IL-8, IL-10 and Leptin in Patients with Chronic Hepatitis B (CHB), Liver Cirrhosis (LC) and Primary Hepatocellular Carcinoma (PHC). J of Radioimmunology, 2012, 25(2): p. 127-130.

[22]Zhang, X., L. Liu and Q. Liu, Relativity of serum leptin level and response to antiviral therapy in patients with chronic hepatitis B. J of Shandong Univ(Health Sciences), 2012. 50(9): p. 96-99.

[23] Su, Y., et al., Serum Leptin Levels in Patients with Chronic Hepatitis B and the Correlation with Hepatic Fibrosis. Acta Acad Med Weifang, 2012, 34(2): p. 96-98.

[24]Jiang, D., X. Tong and D. Yao, Expression and clinical significance of serum leptin in patients with chronic hepatitis B. Jinagsu Med J, 2009. 35(4): p. 400-401.

[25]Li, N., The detection and clinical significance of serum leptin,peripheral B cell number,T cell subsets distribution type in patients with chronic hepatitis B. China Prac Med, 2009. 4(36): p. 15-16.

[26]Zhao, C., X. Wang and X. Wu, Changes of serum leplin levels in patients with chronic hepatitis B. Inter J Epidemiol Infect Dis, 2008. 35(4): p. 236-238.

[27]Guo, X., et al., The relationship between serum leptin and fibrosis in patients with chronic hepatitis B incorporate alcoholic liver disease. Chin J Prim Med Pharm, 2008. 15(9): p. 1461-1462.

[28]Li, H., Y. Wang and Z. Li, the Serum Leptin Level Change and Clinical Significance of Child Hepatitis B Patient. J of Mathematical Med, 2007, 20(3): p. 321-322.

[29]Huang, X. and Y. Lin, Leptin level in patients with chronic hepatitis B and cirrhosis and its correlation with fasting insulin level. J Fourth Mil Med Univ, 2007, 28(10): p. 926-928.

[30] Aşçi, Z., et al., Predictive Value of Serum Fibrosis Markers (Endoglin, Leptin and Immunoglobuline) with Liver Histopathology in Patients with Chronic. Turkiye Klinikleri Journal of Medical Science, 2012, 6(32): p. 1569-1575.

[31]Faggioni, R., K.R. Feingold and C. Grunfeld, Leptin regulation of the immune response and the immunodeficiency of malnutrition. FASEB J, 2001. 15(14): p. 2565-71.

[32] McShane, B.B., U. Bockenholt and K.T. Hansen, Adjusting for Publication Bias in Meta-Analysis: An Evaluation of Selection Methods and Some Cautionary Notes. Perspect Psychol Sci, 2016. 11(5): p. 730-749. 